The Impact of Large-Scale Surveys on Pulsating Star Research

ASP Conference Series, Vol. 203, 2000

L. Szabados \& D. W. Kurtz, eds.

\title{
A Search of Variable Stars in the Tycho Observations
}

\author{
Sandrine Piquard, Jean-Louis Halbwachs \\ Observatoire Astronomique de Strasbourg, 11 rue de l'Université, \\ F-67000 Strasbourg, France
}

Claus Fabricius

Copenhagen University Observatory, Juliane Maries Vej 30, DK-2100

Copenhagen $\emptyset$, Denmark

\author{
Ralf Geckeler \\ Institute for Astrophysics, Technikerstrasse 25, A-6020 Innsbruck, \\ Austria
}

Andreas Wicenec

European Southern Observatory, Karl-Schwarzschild-Strasse 2, D-85748 Garching, Germany

\begin{abstract}
The selection of variable stars in the Tycho catalogue was re-considered. A method based on a Kolmogorov-Smirnov test was elaborated, giving 729 candidate variable stars. This number could increase to about 1000 in the future.
\end{abstract}

\section{A Search for Variable Stars in the Tycho Observations}

The Tycho catalogue (ESA 1997) was based on the observations of the star mapper of the Hipparcos satellite. It contains the positions and the magnitudes (in the photometric bandpasses $B_{T}$ and $V_{T}$ ) of about 1 million stars. A variability flag was included, but it was assigned only to stars in the bright half of the catalogue, and it was questionable already for stars between 8 and 9 mag. For these reasons, a new method of variability detection was set up specially for the Tycho observations. It consisted in a preparation of the data in order to increase their reliability, and in a statistical treatment based on the Kolmogorov-Smirnov test, as explained hereafter.

First at all, the measurements were corrected for systematic errors (Halbwach 1997). After that, the consecutive transits detected in 20 min were compared among themselves, and the bright outliers generated by light contamination from background stars were rejected. The remaining transits were gathered in one aggregate observation. The $B_{T}$ and the $V_{T}$ bandpasses were merged and only the magnitude in the resulting $T$ photometric band was considered.

The next step was the calculation of the probabilities to obtain $T$ magnitudes fainter than the ones found in each aggregate measurement. For that purpose, it was assumed that the stars were constant in brightness, and that 
their $B_{T}$ and $V_{T}$ magnitudes were exactly the mean magnitudes provided by the Tycho catalogue. The condition that each detected transit had necessarily a signal-to-noise ratio larger than the detection threshold was taken into account in the computation. The probabilities thus derived were considered in a variant of the Kolmogorov-Smirnov test, and the stars having aggregate measurements abnormally scattered were selected. This method was preferred to the usual variance analysis test, since the acquisition process of Tycho data did not produce magnitude measurements obeying a Gaussian distribution (Friedrich, König, \& Wicenec 1997).

\section{A Selection of Candidate Variable Stars}

Our method was applied to 760390 stars from the Tycho Epoch Photometry Database, and 729 candidate variable stars were detected at the $0.02 \%$ level of significance. The number of false alarms was estimated about to be $760390 \times$ $0.0002=152$, and their proportion among our selection should be $152 / 729=$ 20\%. 347 of these stars were already quoted as variable in GCVS, NSV, or Hipparcos. When known, their variability types are mainly semi-regular (64), eclipsing binary (61), irregular (37), $\delta$ Cep (36), Mira (33), and RR Lyr (10). The known periodic variables were used to prepare the light curve analysis of the 382 candidate new variable stars in the list (Andronov, Cuypers, \& Piquard 2000).

\section{Future Prospects}

The same method will be applied assuming better estimations of the mean magnitudes of the stars. The magnitudes derived in the second processing of the Tycho data (Høg et al. 1998) were used in an assessment: it appeared that the number of candidate variable stars could thus increase to about 1000 .

\section{References}

Andronov, I. L., Cuypers, J., \& Piquard, S. 2000, in these proceedings, p. 64 ESA 1997, The Hipparcos and Tycho Catalogues, ESA SP-1200

Friederich, S., König, M., \& Wicenec, A. 1997, in Hipparcos Venice'97, ed. B. Battrick, ESA SP-402, 441

Halbwachs, J.-L. 1997, in The Hipparcos and Tycho Catalogues, ESA SP-1200, vol. 4 , ch. 9

Høg, E., Fabricius, C., Makarov, V. V., Bastian, U., Schwekendiek, P., Wicenec, A. 1999, in Modern Astrometry and Astrodynamics, ed. R. Dvorak, H. F. Haupt, \& K. Wodnar (Vienna: Austrian Academy of Sciences), 43 